\title{
Dried blood spots are an efficient blood sampling method for the detection of SARS-CoV-2 antibodies [version 1; peer
}

\section{review: 2 not approved]}

\author{
Juan Carlos Cassano, Michael Reut, Wolfgang Korte
}

Zentrum für Labormedizin, Frohbergstrasse 3, St Gallen, 9001, Switzerland

\author{
V1 First published: 20 Nov 2020, 9:1354 \\ https://doi.org/10.12688/f1000research.25836.1 \\ Latest published: 20 Nov 2020, 9:1354 \\ https://doi.org/10.12688/f1000research.25836.1
}

\section{Abstract}

A novel coronavirus termed SARS-CoV-2 caused an outbreak in December of 2019 which has led to pandemic. Currently several serological diagnostic assays exist for the detection of SARS-CoV-2, which require the collecting of whole blood that brings about problems including the invasive nature of venepuncture, poor acceptance by patients and their storage and transportation. A more fast, efficient and less tedious method that allows mass blood sampling is necessary during a pandemic to quickly diagnose disease and obtain population serological data.

Dried blood spot (DBS) sampling has been used for several decades for the accurate detection of viral specific antibodies and remains one the most convenient methods for obtaining serological data on exposed patients. Here we evaluate the use of DBS sampling on current viral serological assays including SARS-CoV-2. DBS samples were collected from six patients (five control and one positive for SARS-CoV-2 infection) and patient serum was extracted and tested blindly using commercially available antibody test kits for Coxiella burnetti, parvovirus B19 and SARS-CoV-2.

The results demonstrate that antibodies recovered from DBS after elution are comparable to those found in serum, indicating that serological tests can be adapted to test DBS samples from patients using our modified protocol. Because DBS sampling is a much faster and cheaper method of sampling blood, this modification could therefore allow for potential nationwide testing for epidemiological studies.

\section{Keywords}

SARS-CoV-2, Coronavirus, Dried Blood Spot, Blood, Patient, Serology, Sampling, Antibody

\section{Open Peer Review \\ Approval Status $\times \times$ \\ 12 \\ version 1 \\ 20 Nov 2020

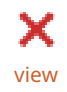

1. Livia Villar, FIOCRUZ (Oswaldo Cruz

Foundation), Rio de Janeiro, Brazil

2. Davor Brinc (iD), University of Toronto,

Toronto, Canada

Any reports and responses or comments on the article can be found at the end of the article. 


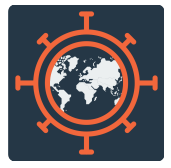

This article is included in the Emerging Diseases

and Outbreaks gateway.

collection.

\section{Corresponding author: Juan Carlos Cassano (JuanCarlos.Cassano@zlmsg.ch)}

Author roles: Cassano JC: Formal Analysis, Investigation, Methodology, Project Administration, Supervision, Validation, Visualization, Writing - Original Draft Preparation, Writing - Review \& Editing; Reut M: Investigation, Validation; Korte W: Conceptualization, Project Administration, Resources, Supervision, Writing - Original Draft Preparation, Writing - Review \& Editing

Competing interests: No competing interests were disclosed.

Grant information: The author(s) declared that no grants were involved in supporting this work.

Copyright: ๑ 2020 Cassano JC et al. This is an open access article distributed under the terms of the Creative Commons Attribution License, which permits unrestricted use, distribution, and reproduction in any medium, provided the original work is properly cited.

How to cite this article: Cassano JC, Reut M and Korte W. Dried blood spots are an efficient blood sampling method for the detection of SARS-CoV-2 antibodies [version 1; peer review: 2 not approved] F1000Research 2020, 9:1354 https://doi.org/10.12688/f1000research.25836.1

First published: 20 Nov 2020, 9:1354 https://doi.org/10.12688/f1000research.25836.1 


\section{Introduction}

Coronavirus disease 2019 (COVID-19) was first reported in December, 2019 in Wuhan, China and on March 112020 it was declared a pandemic by the World Health Organization. COVID-19 is caused by severe acute respiratory syndrome coronavirus 2 (SARS-CoV-2) which shares up to $82 \%$ genome identity with SARS-CoV, which first caused an outbreak in $2003^{1}$. It is characterized by fever, cough, malaise, shortness of breath and in severe cases pneumonia ${ }^{2}$.

Reliable serological data are in urgent need to guide treatment, infection control, epidemiological measures and vaccination. Whilst several serological assays are now available for antibody detection in the form of anti-SARS-CoV-2 enzymelinked immunosorbent assay (ELISA) kits, most of these require the collection of serum from whole blood. Whole blood collection can pose several problems in their collection, storage and transportation. The collection of whole blood requires venepuncture, which is an invasive procedure and can be poorly accepted by patients. Equipment requirements for the isolation of serum include syringes, tubes, centrifuges, refrigerators and freezers. After collection, whole blood requires maintenance at the site of collection with refrigerated storage and transportation from distant locations ${ }^{3}$.

Dried blood spot (DBS) sampling offers practical, clinical, and financial advantages as compared to conventional blood testing. Compared with standard venepuncture, the fingerstick or heelpricking sampling of capillary blood is easy to perform and relatively painless. It can be carried out by patients at home without a trained health care worker or medical laboratory, which is of particular advantage in settings with limited infrastructure. Utilizing capillary blood requires less sampling volume, negates the use of centrifuges or even basic laboratory equipment, and transportation is facilitated as it does not require refrigeration or high skill preventing contamination ${ }^{4}$.

DBS has been used for the detection of antibodies to several viral disease including rubella, HIV, measles and hepatitis and has shown good correlation to serum samples when assayed with commercially available kits $^{5-9}$. Further, DBS sampling allows for the detection of different immunoglobulin isotypes IgG, IgA, $\operatorname{IgE}$ and $\operatorname{IgM}^{5-8,10}$. For this reason, we decided to evaluate the use of DBS sampling on current viral serological assays for SARS-CoV-2. DBS samples were collected from six patients (five control and one positive for SARS-CoV-2 infection) and patient serum was eluted and tested blindly using commercially available antibody test kits for Coxiella burnetti, parvovirus B19 and SARS-CoV-2.

Our results demonstrate that antibodies recovered from DBS after elution are comparable to those found in serum, indicating that commercially available serological assays can be adapted to test DBS samples from patients using our modified protocol. Adapting ELISA assays to DBS sampling may facilitate potential nationwide testing for epidemiological studies into SARS-CoV-2.

\section{Methods}

\section{Ethical statement}

According to Swiss legislation, the Human Research Act is applicable to research on human diseases and on the structure and function of the human body. As this project is a quality control on the blood samples of six participants, it does not fall under the scope of research under Article $3 \mathrm{HFG}$ and an assessment by an ethics committee was therefore deemed unnecessary by the Ethics Committee Of Eastern Switzerland. All patients gave both oral and written consent to participate in this study.

\section{DBS sampling}

Potential participants were identified within our institute and selected based on their medical history. DBS samples were collected by a medical laboratory technician from six adult patients (five control and one SARS-CoV-2 positive, as confirmed by PCR and antibody testing). Blood was collected through skin puncture twice over two separate weeks. Briefly, the skin was cleaned on the palmar side of the 4th finger on the left hand with $70 \%$ isopropyl alcohol. The skin was punctured using a single-use safety BD Microtainer contact activated lancet (Becton Dickinson, Dublin, Ireland) and the first drop of blood was wiped off. Blood was collected on to a Whatman 903 Protein Saver Card (GE Healthcare, UK). DBS were allowed to dry completely overnight at room temperature. Using a single-use $6 \mathrm{~mm}$ biopsy punch (GSK Consumer Healthcare, UK), one spot was punched out from each DBS and transferred into a well of a 96-well plate using a single-use disposable tweezer (Megro, Wesel, Germany). This process was repeated for all punched spots. To elute serum, $250 \mu \mathrm{l}$ elution buffer (1 X PBS, $0.05 \%$ Tween $20,0.1 \%$ BSA) was placed in each well and the 96-well plate was placed on a rotator plate overnight at RT. Eluates were then collected and stored at $4{ }^{\circ} \mathrm{C}$ for ELISA.

\section{ELISA assay}

DBS eluates were subjected to the following commercial immunoassay kits: Parvovirus B19 IgG (Kit number ESR122G, Serion Diagnostic, Germany), Coxiella burnetii Phase 2 IgG (Kit number ESR1312G, Serion Diagnostics), Anti-SARS-CoV-2ELISA IgG and Anti-SARS-CoV-2-ELISA IgA (Kit numbers EI 2606-9601 G and EI 2606-9601 A, Euroimmun, Lübeck, Germany) and the EDI ${ }^{\mathrm{TM}}$ Novel Coronavirus COVID-19 IgG ELISA kit (Kit number KT-1032, Epitope Diagnostics, San Diego, USA) to detect the presence of antibodies. Because seroprevalence is high for parvovirus ${ }^{11}$ and low for Coxiella burnetii in Europe ${ }^{12,13}$, the parvovirus B19 IgG and Coxiella burnetti Phase 2 IgG ELISAs acted as positive and negative controls, respectively. All the listed commercial immunoassays in our study require a minimum of $10 \mu \mathrm{l}$ serum sample to be diluted in a 1:100 dilution ratio with sample diluent at a final volume of $1000 \mu \mathrm{l}$. It has been reported that a $6 \mathrm{~mm}$ punched spot contains approximately $5 \mu \mathrm{l}$ of serum ${ }^{5}$. Therefore, sample preparation for the commercial ELISA required $2 \times 250 \mu \mathrm{l}$ patient sample eluates to be pooled $(500 \mu \mathrm{l})$, to which $500 \mu \mathrm{l}$ of sample diluent was added to make a final volume of $1000 \mu \mathrm{l}$.

The commercial ELISAs were run on a DSX® Automated ELISA Processing System (Dynex Technolgies, Denkendorf, 
Germany) according to the manufacturer's instructions. A total of two DBS samples per patient was run for each commercial ELISA over two separate weeks. OD values for each DBS sample were determined at $\lambda=405 \mathrm{~nm}(\lambda=405 \mathrm{~nm}$ reference wavelength) for Coxiella burnetii, $\lambda=405 \mathrm{~nm}(\lambda=650 \mathrm{~nm}$ reference wavelength) for parvovirus $\mathrm{B} 19, \lambda=450 \mathrm{~nm}(\lambda=$ $620 \mathrm{~nm}$ reference wavelength) for both anti-SARS-CoV-2 IgG and $\operatorname{IgA}$, and $\lambda=450 \mathrm{~nm}$ ( $\lambda=450 \mathrm{~nm}$ reference wavelength) for $\mathrm{EDI}^{\mathrm{TM}}$ Novel Coronavirus COVID-19 IgG.

\section{Data analysis}

ELISA assays were performed on the DYNEX DSX, and analysed on DSXLab software (version 640.2, DYNEX Technologies GmbH, Denkendorf, Germany). OD values for all DBS samples were corrected using the average control serum sample giving a ratio, as described by the manufacturer. For the Coxiella burnetti ELISA, DBS samples registering a ratio of $>1.1$ were considered positive results, whilst ratios of $<0.9$ indicated negative results. For the parvovirus B19 ELISA, DBS samples registering a ratio of 5.01 indicated positive results whilst those registering a ratio of $<2.99$ were considered negative results. For the commercial anti-SARS-CoV-2 IgG and IgA ELISA kits from Euroimmun, DBS samples registering ratios of $>1.1$ indicated positive results, ratios of $<0.8$ indicated negative results, whilst ratios between 0.8 and 1.1 indicated borderline results. For the commercial EDI ${ }^{\mathrm{TM}}$ Novel Coronavirus COVID-19 IgG ELISA kit, DBS samples that resulted in ratios $>1.1$ indicated positive results, ratios of $<0.9$ indicated negative results, whilst ratios between 0.9 and 1.1 indicated borderline results.

\section{Results}

\section{Elution of DBS samples}

The elution buffer ( $1 \mathrm{X}$ PBS, $0.05 \%$ Tween $20,0.1 \%$ BSA) was found to be effective at eluting serum from DBS samples. Because each $6 \mathrm{~mm}$ punched spot contains approximately $5 \mu \mathrm{l}$ of serum, and elution with $250 \mu \mathrm{l}$ represented a 1:50 dilution of the DBS sample.

\section{ELISA with DBS samples}

All six patient (A-F) DBS samples were tested for antibodies using the commercial ELISA kits parvovirus B19 (IgG), Coxiella burnetii Phase 2 (IgG), anti-SARS-CoV-2-ELISA (IgG and IgA) and the EDI $^{\mathrm{TM}}$ novel coronavirus COVID-19 (IgG) ELISA kit. All DBS samples were positive for parvovirus B19 IgG antibodies, whilst negative for Coxiella burnetti $\operatorname{IgG}$ as expected (Table 1). DBS samples assayed on both the SARS-CoV-2 IgG from Euroimmun and Epitope Diagnostics were negative in control patients (A-E), whilst positive for the single patient (F) with a previous history of SARS-CoV-2 infection (Table 2, Table 3).

The ELISA for $\operatorname{Ig} \mathrm{A}$ antibodies from Euroimmun indicated that the DBS sample from the SARS-CoV-2 patient (F) was positive for specific IgA antibodies (Table 2). Interestingly, several DBS samples from control patients displayed borderline positive results, whilst one DBS sample from one patient (E) with no apparent history of exposure to SARS-CoV-2 showed the presence of IgA antibodies (Table 2).

\section{Discussion}

The results that we have obtained serve to confirm the effectiveness of using DBS samples for the serological detection of antibodies, whilst extending the applicability of current commercial immunoassays for the detection of SARS-CoV-2. Our results are in accordance with the expected seroprevalence of Coxiella burnetii and parvovirus in Europe ${ }^{11-13}$. Of importance is that we were able to detect both $\operatorname{IgG}$ and $\operatorname{IgA}$ antibodies in the patient with a history of SARS-CoV-2 infection using the commercially available kits. This indicates that DBS sampling may be as reliable as serum sampling via venepuncture for the detection of antibodies to SARS-CoV-2. Although $\operatorname{IgA}$ was detected as both borderline and positive results in asymptomatic control patients which were thought to be negative for exposure to SARS-CoV-2, this observation may be due to several reasons. Borderline cross-reactivity has been reported for the anti-SARS-CoV-2-ELISA (IgA) from Euroimmun ${ }^{14}$. Samples

Table 1. Results obtained for the detection of antibodies in dried blood spots (DBS) from the commercial Coxiella burnetti and parvovirus B19 ELISA kits. Calculated ratios were used to determine serological status. For the Coxiella burnetti ELISA, positive results (pos) indicate a ratio $>1.1$, negative results (neg) indicate a ratio $<0.9$. For the parvovirus B19 ELISA, positive results (pos) indicate a ratio $>5.01$, negative results (neg) indicate a ratio $<2.99$. For each patient, DBS samples were taken and measured over two separate weeks.

\begin{tabular}{|c|c|c|c|c|c|c|c|c|c|c|c|c|c|}
\hline \multirow{2}{*}{\multicolumn{2}{|c|}{ Test }} & \multicolumn{12}{|c|}{ Patient } \\
\hline & & \multicolumn{2}{|l|}{ A } & \multicolumn{2}{|l|}{ B } & \multicolumn{2}{|l|}{ C } & \multicolumn{2}{|l|}{ D } & \multicolumn{2}{|l|}{$\mathbf{E}$} & \multicolumn{2}{|l|}{$\mathbf{F}$} \\
\hline \multirow[t]{3}{*}{ Coxiella } & O.D. & 0.131 & 0.173 & 0.096 & 0.105 & 0.143 & 0.127 & 0.163 & 0.194 & 0.157 & 0.167 & 0.168 & 0.211 \\
\hline & Ratio & 0.3 & 0.4 & 0.2 & 0.2 & 0.3 & 0.3 & 0.3 & 0.4 & 0.3 & 0.4 & 0.3 & 0.5 \\
\hline & Result & neg & neg & neg & neg & neg & neg & neg & neg & neg & neg & neg & neg \\
\hline \multirow[t]{3}{*}{ Parvovirus } & O.D. & 1.584 & 1.553 & 0.978 & 0.902 & 1.755 & 1.535 & 0.841 & 0.901 & 1.763 & 1.553 & 1.805 & 1.553 \\
\hline & Ratio & 142.2 & 150 & 22.4 & 26.4 & 150 & 150 & 16.3 & 26.3 & 150 & 150 & 150 & 150 \\
\hline & Result & pos & pos & pos & pos & pos & pos & pos & pos & pos & pos & pos & pos \\
\hline
\end{tabular}


Table 2. Results obtained for the detection of SARS-CoV-2 IgG and IgA antibodies in dried blood spots (DBS) from the Euroimmun commercial anti-SARS-CoV-2 ELISA kits. Calculated ratios were used to determine serological status. For both the SARS-CoV-2 IgG and IgA ELISA, positive results (pos) indicate a ratio $>1.1$, negative results (neg) indicate a ratio $<0.8$. Borderline results $(\mathrm{gw}$ ) indicate a ratio between 0.8 and 1.1. For each patient, DBS samples were taken and measured over two separate weeks.

\begin{tabular}{|c|c|c|c|c|c|c|c|c|c|c|c|c|c|}
\hline \multirow{2}{*}{\multicolumn{2}{|c|}{ Test }} & \multicolumn{12}{|c|}{ Patient } \\
\hline & & \multicolumn{2}{|l|}{ A } & \multicolumn{2}{|l|}{ B } & \multicolumn{2}{|l|}{ C } & \multicolumn{2}{|l|}{ D } & \multicolumn{2}{|l|}{$\mathbf{E}$} & \multicolumn{2}{|l|}{$\mathbf{F}$} \\
\hline \multirow[t]{3}{*}{ SARS-CoV-2 IgG } & O.D. & 0.135 & 0.206 & 0.101 & 0.194 & 0.097 & 0.148 & 0.113 & 0.178 & 0.102 & 0.188 & 1.249 & 2.084 \\
\hline & Ratio & 0.543 & 0.735 & 0.408 & 0.691 & 0.389 & 0.529 & 0.456 & 0.635 & 0.409 & 0.669 & 5.025 & 7.425 \\
\hline & Result & neg & neg & neg & neg & neg & neg & neg & neg & neg & neg & pos & pos \\
\hline \multirow[t]{3}{*}{ SARS-CoV-2 IgA } & O.D. & 0.322 & 0.367 & 0.18 & 0.265 & 0.259 & 0.205 & 0.154 & 0.417 & 0.894 & 1.24 & 3.135 & 3.15 \\
\hline & Ratio & 1.07 & 1.276 & 0.599 & 0.922 & 0.859 & 0.713 & 0.512 & 1.451 & 2.967 & 4.315 & 10.405 & 10.966 \\
\hline & Result & GW & pos & neg & gw & GW & neg & neg & pos & pos & pos & pos & pos \\
\hline
\end{tabular}

Table 3. Results obtained for the detection of SARS-CoV-2 IgG antibodies in dried blood spots (DBS) from the Epitope Diagnostics commercial EDI ${ }^{\mathrm{TM}}$ Novel Coronavirus COVID-19 IgG ELISA kit. Calculated ratios were used to determine serological status. Positive results (pos) indicate a ratio $>1.1$, negative results (neg) indicate a ratio <0.9. Borderline results (gw) indicate a ratio between 0.9 and 1.1. For each patient, DBS samples were taken and measured over two separate weeks.

\begin{tabular}{|c|c|c|c|c|c|c|c|c|c|c|c|c|c|}
\hline \multirow{2}{*}{\multicolumn{2}{|c|}{ Test }} & \multicolumn{12}{|c|}{ Patient } \\
\hline & & \multicolumn{2}{|l|}{ A } & \multicolumn{2}{|l|}{ B } & \multicolumn{2}{|l|}{ C } & \multicolumn{2}{|l|}{ D } & \multicolumn{2}{|l|}{$\mathbf{E}$} & \multicolumn{2}{|l|}{$\mathbf{F}$} \\
\hline \multirow[t]{3}{*}{ SARS-CoV-2 IgG } & O.D. & 0.159 & 0.185 & 0.129 & 0.126 & 0.151 & 0.126 & 0.191 & 0.186 & 0.164 & 0.195 & 0.503 & 0.548 \\
\hline & Ratio & 0.5 & 0.6 & 0.41 & 0.41 & 0.48 & 0.41 & 0.6 & 0.61 & 0.52 & 0.64 & 1.59 & 1.79 \\
\hline & Result & neg & neg & neg & neg & neg & neg & neg & neg & neg & neg & pos & pos \\
\hline
\end{tabular}

negative for $\operatorname{IgG}$ and borderline or positive for $\operatorname{IgA}$ may also indicate prior exposure to SARS-CoV-2.

The advantages of adapting commercial immunoassays to DBS are many. Rapid diagnostic testing is necessary in limitedresource settings. DBS offers an affordable alternative to venepuncture. Because little training is required to sample blood using the DBS method, a greater number of the population can be sampled for epidemiological studies. DBS samples can be dried and stored at room temperature for several weeks, they require little storage space and can even be sent to testing sites via mail. Such sampling may be especially suitable in serological screening programs in developing countries, where SARS-CoV-2 is having its greatest impact. DBS sampling may further facilitate widespread sampling of capillary blood, enabling analysis with high-throughput laboratory-based immunoassays, which would allow rapid nationwide serological screening that is of absolute necessity for disease control during a pandemic.

The main disadvantage of DBS sampling is that most of the existing commercial immunoassays have not been validated or received the necessary regulatory approval for DBS sampling to be implemented. Despite this drawback, DBS sampling has shown promise, in that it has been successfully used to detect the presence of antibodies to several disease-causing viral pathogens including rubella, HIV, measles and hepatitis ${ }^{5-9}$. A meta-analysis on the diagnostic accuracy of HIV-antibody and HBV- surface antigen from DBS samples compared to venous blood samples was associated with excellent diagnostic accuracy ${ }^{9}$. However, the study found that the lack of standardization of sampling, handling, processing, storage and transportation processes limits their use. The use of DBS sampling for routine or high-throughput serological assays would therefore require a standardized method for the pre-analytical treatment of specimens and processing of the sample and subsequent validation with commercial assays.

Several commercially available automated biopsy punch instruments exist that would facilitate the processing of DBS samples for high-throughput immunoassay application. Coupled with automated immunoassays platforms, automated biopsy punch instruments could facilitate the standardization of DBS technology and bring us one step closer to adapting DBS sampling to routine immunoassays. What remains a challenge 
is the standardization of the sampling protocol, the storage and transportation to a facility that would combine such a set-up.

\section{Conclusions}

DBS sampling offers a promising alternative to serum sampling via venepuncture and can accurately detect antibodies to SARS-CoV-2. DBS sampling could therefore be adapted for future serological screening of large populations. Lack of standardization of sampling and testing limits the wider application of DBS.

\section{Data availability}

All data underlying the results are available as part of the article and no additional source data are required.
1. Chan JFW, Kok KH, Zhu Z, et al.: Genomic characterization of the 2019 novel human-pathogenic coronavirus isolated from a patient with atypical pneumonia after visiting Wuhan. Emerg Microbes Infect. 2020; 9(1): 221-36. PubMed Abstract | Publisher Full Text | Free Full Text

2. Huang C, Wang Y, Li X, et al.: Clinical features of patients infected with 2019 novel coronavirus in Wuhan, China. Lancet. 2020; 395(10223): 497-506. PubMed Abstract | Publisher Full Text | Free Full Text

3. Bellini WJ, Helfand RF: The challenges and strategies for laboratory diagnosis of measles in an international setting.J Infect Dis. 2020; 187(Suppl.1): S283-S290.

PubMed Abstract | Publisher Full Text

4. Snijdewind IJM, van Kampen JJA, Fraaij PLA, et al.: Current and future applications of dried blood spots in viral disease management. Antiviral Res. 2012; 93(3): 309-21.

PubMed Abstract | Publisher Full Text

5. Lillo F, Varnier OE, Mantia E, et al.: Detection of HIV-1 antibodies in blood specimens spotted on filter-paper. Bull World Health Organ. 1992; 70(3): 323-326.

PubMed Abstract | Free Full Text

6. Riddell MA, Byrnes GB, Leydon JA, et al.: Dried venous blood samples for the detection and quantification of measles IgG using a commercial enzyme immunoassay. Bull World Health Organ. 2003; 81(10): 701-7. PubMed Abstract | Free Full Text

7. Mercader S, Featherstone D, Bellini WJ: Comparison of available methods to elute serum from dried blood spot samples for measles serology. J Virol Methods. 2006; 137(1): 140-9.

PubMed Abstract | Publisher Full Text

8. Helfand RF, Cabezas C, Abernathy E, et al.: Dried blood spots versus sera for detection of rubella virus-specific immunoglobin M (IgM) and IgG in samples collected during a rubella outbreak in Peru. Clin Vaccine Immunl. 2007; 14(11): 1522-5.

PubMed Abstract | Publisher Full Text | Free Full Text

9. Lange B, Cohn J, Roberts T, et al.: Diagnostic accuracy of serological diagnosis of hepatitis $C$ and $B$ using dried blood spot samples (DBS): two systematic reviews and meta-analyses. BMC Infect Dis. 2017; 17(Suppl 1): 700. PubMed Abstract | Publisher Full Text | Free Full Text

10. Andersen NJ, Mondal TK, Preissler MT, et al.: Detection of immunoglobulin isotypes from dried blood spots. J Immunol Methods. 2014; 404: 24-32. PubMed Abstract | Publisher Full Text | Free Full Text

11. Mossong J, Hens N, Friedrichs V, et al.: Parvovirus B19 infection in five european countires; seroepidemiology, force of infection and maternal risk of infection. Epidemiol Infect. 2008; 136(8): 1059-68. PubMed Abstract | Publisher Full Text | Free Full Text

12. Tobudic S, Nedomansky K, Poeppl W, et al.: Seroprevalence for Coxiella burnetii, Francisella tularensis, Brucell abortus and Brucell Melitensis in Austrian adults: A cross- sectional survey among military personnel and civilians. Ticks Tick Borne Dis. 2014; 5(3): 315-7. PubMed Abstract | Publisher Full Text

13. van Roeden SE, Holsboer EW, Oosterheert It, et al: Seroprevalence of Coxiella burnetii antibodies and chronic $\mathrm{Q}$ fever among post-mortal and living donors of tissues and cells from 2010 to 2015 in the netherlands. Euro Surveill. 2018; 23(9): 17-00384. PubMed Abstract | Publisher Full Text | Free Full Text

14. Beavis KG, Matushek SM, Abeleda APF, et al.: Evaluation of the EUROIMMUN Anti-SARS-CoV-2 ELISA assay for detection of IgA and IgG antibodies. J Clin Virol. 2020; 129: 104468

PubMed Abstract | Publisher Full Text | Free Full Text 


\section{Open Peer Review}

\section{Current Peer Review Status: $\mathrm{X} X$}

\section{Version 1}

Reviewer Report 11 August 2021

https://doi.org/10.5256/f1000research.28513.r90319

(C) 2021 Brinc D. This is an open access peer review report distributed under the terms of the Creative Commons Attribution License, which permits unrestricted use, distribution, and reproduction in any medium, provided the original work is properly cited.

\section{Davor Brinc}

Department of Laboratory Medicine and Pathobiology, University of Toronto, Toronto, Ontario, Canada

\section{General comments}

The manuscript reports a study of antibody detection against SARS-CoV-2 using DBS samples. Use of DBS for testing different analytes in blood, including testing of infectious diseases and serology, is an important and developing field. The topic is therefore relevant and would be of wide interest.

The main issue with reported study is very low $n$ value; there is only one patient positive for SARSCoV-2 which makes it difficult to draw conclusions about the performance of the particular methodology used in the study.

\section{Specific comments:}

Method section:

Patient selection is described as based on PCR and antibody testing. More details need to be given; which PCR test was used (which specific test, manufacturer, Ct/viral load if possible) on which sample type; which antibody testing (isotype, manufacturer, antigen) on which sample type. Also days since symptom onset relative to PCR and antibody testing as well as symptom description (mild/severe/hospitalized) are needed.

For DBS sampling, days since symptom onset and days since PCR and antibody testing sample collection are needed. More details about DBS elution would be helpful, for example, how many circles on the filter paper were spotted, how many total punches were made and then transferred to 96 well plate. Was 250 uL elution buffer used for one $6 \mathrm{~mm}$ punch, this seems to be suggested in the Results section, but perhaps could be described more clearly in the Methods section. For the cutoffs described for ELISAs, are the cutoffs the same as for plasma/serum as suggested by the manufacturer? Source of cutoffs should be clearly given.

\section{Results section}

For SARS-CoV-2 DBS results, comparison of signals obtained from corresponding matched 
plasma/serum using the same assays would be helpful.

For control samples showing IgA positivity, knowing the corresponding result from plasma/serum would be helpful.

\section{Discussion section}

There are several published studies of DBS serology for SARS-CoV-2 using in-house developed ELISA, commercial ELISA, and commercial automated analyzers, including Euroimmun.

Euroimmun also has specific dried blood spot application.

Clinical performance of DBS for SARS-CoV2 detection (e.g. sensitivity, specificity) reported by such studies should be discussed.

Detection of IgA in control samples should be discussed in more detail based on previous Euroimmun assay performance. Specificity of Euroimmun assay (both IgG and IgA) based on published studies should also be discussed as any limited specificity could affect data interpretation.

In conclusion, while DBS results from the investigated COVID19+ patient does show likely ability of the proposed DBS methodology to detect positive serology, and this is in agreement with other DBS studies, it is difficult to make more general comments about the performance due to low $\mathrm{n}$ value.

At this stage, the study is basically a case report.

Is the work clearly and accurately presented and does it cite the current literature? Partly

Is the study design appropriate and is the work technically sound?

Partly

Are sufficient details of methods and analysis provided to allow replication by others? Partly

If applicable, is the statistical analysis and its interpretation appropriate?

Not applicable

Are all the source data underlying the results available to ensure full reproducibility? Yes

Are the conclusions drawn adequately supported by the results?

No

Competing Interests: No competing interests were disclosed.

Reviewer Expertise: Clinical chemistry 
I confirm that I have read this submission and believe that I have an appropriate level of expertise to state that I do not consider it to be of an acceptable scientific standard, for reasons outlined above.

Reviewer Report 15 January 2021

https://doi.org/10.5256/f1000research.28513.r75137

(C) 2021 Villar L. This is an open access peer review report distributed under the terms of the Creative Commons Attribution License, which permits unrestricted use, distribution, and reproduction in any medium, provided the original work is properly cited.

Livia Villar

Laboratory of Viral Hepatitis, Oswaldo Cruz Institute, FIOCRUZ (Oswaldo Cruz Foundation), Rio de Janeiro, Brazil

The paper describes a method for detecting SARS CoV-2 antibodies in dried blood spot samples.

There are some issues that is not clear:

Why include Parvovirus and Coxiella testing in the paper?

Authors informed that there are $5 \mathrm{ul}$ of serum in each $6 \mathrm{~mm}$ of paper disc, however the paper cited as reference indicates that $5 \mathrm{~mm}$ of paper disc contains $10 \mathrm{ul}$ of serum. In addition, variation could happen according hematocrit.

The major issue is the few number of samples included for analysis since just one individual presented IgG anti-SARS CoV-2, for example. More samples should be tested to confirm the effectiveness of using DBS samples for the serological detection of SARS CoV-2.

Is the work clearly and accurately presented and does it cite the current literature? Yes

Is the study design appropriate and is the work technically sound?

Partly

Are sufficient details of methods and analysis provided to allow replication by others? Partly

If applicable, is the statistical analysis and its interpretation appropriate? Partly

Are all the source data underlying the results available to ensure full reproducibility? Partly

Are the conclusions drawn adequately supported by the results? 
Partly

Competing Interests: No competing interests were disclosed.

Reviewer Expertise: Virology

I confirm that I have read this submission and believe that I have an appropriate level of expertise to state that I do not consider it to be of an acceptable scientific standard, for reasons outlined above.

The benefits of publishing with F1000Research:

- Your article is published within days, with no editorial bias

- You can publish traditional articles, null/negative results, case reports, data notes and more

- The peer review process is transparent and collaborative

- Your article is indexed in PubMed after passing peer review

- Dedicated customer support at every stage

For pre-submission enquiries, contact research@f1000.com 